\title{
EL VALOR DEL ESTUDIO DE LA FILOSOFÍA ANTIGUA (A MODO DE PRESENTACIÓN)
}

\author{
Gabriela Rossi ${ }^{1}$ \\ Universidad Adolfo Ibáñez (Chile)
}

Recibido: 01.12.2018 - Aceptado: 11.12.2018

\begin{abstract}
RESUMEN
En estas páginas ofrezco, en primer lugar, algunas consideraciones respecto del valor filosófico del estudio de la filosofía antigua, tanto en vista de los aspectos metodológicos y argumentativos desplegados por los textos de los filósofos antiguos, como en atención al contenido de estos textos. En este último sentido, recojo algunas de las muchas instancias de rehabilitación de filósofos y doctrinas antiguas que han tenido lugar a lo largo de los últimos decenios para poner de relieve cómo y hasta qué punto la reflexión filosófica sustantiva no se desentiende de hecho del estudio de los antiguos, sino que más bien encuentra en ellos, una y otra vez, fuente de inspiración filosófica. Por último, realizo un breve recorrido por los artículos que componen este número monográfico.
\end{abstract}

Palabras clave: Filosofía antigua; historia de la filosofía; actualidad; rehabilitación; filosofía.

\begin{abstract}
In these pages I offer, in the first place, some general reflections on the philosophical value of the study of Ancient Philosophy. I consider both the interest of the methodological and argumentative aspects of ancient texts, and the relevance of their content. Regarding the last point, I review some instances of "rehabilitation" of ancient philosophers during the last decades, in order to highlight the manner and extent to which creative philosophical reflection pays heed to the ancients and finds in them, once and again, philosophical inspiration and a source of fresh answers to old philosophical problems. Finally, I summarize the content of the articles within this special issue.
\end{abstract}

Keywords: Ancient philosophy; history of philosophy; actuality; rehabilitation; philosophy.

\footnotetext{
${ }^{1}$ rossigabriela@gmail.com

gabriela.rossi@uai.cl
} 
Este número monográfico fue concebido por vez primera hacia mediados del año 2017. En aquel entonces, propuse a la revista Síntesis oficiar como editora invitada de un volumen especial sobre Filosofía Antigua, con la idea de plasmar en él parte de la intensa actividad de alta calidad académica de estudiosos jóvenes y consagrados en el área. Por circunstancias que han sido, en parte, azarosas, en el lapso que ha transcurrido hasta ahora he debido asumir la tarea de dirigir la revista. La accidental superposición de papeles que me toca jugar en el momento en que este número ve la luz me obliga a advertir que no escribo estas páginas como directora de Síntesis, sino como editora de este volumen especial. $^{2}$

Es sin duda un desafío introducir un conjunto de textos de especialistas en Filosofía Antigua para una revista académica dedicada a la filosofía en general. Quienes nos dedicamos a este período sabemos que existen algunos prejuicios extendidos, más o menos fundados según el caso, respecto del estudio de los filósofos de la Antigüedad, que nos obligan a veces a ensayar algún tipo de justificación respecto de la pertinencia y la necesidad de dedicar nuestros esfuerzos a textos que fueron escritos hace dos mil años, y más. Es cierto que, muchas veces, quienes se dedican a textos y filósofos más actuales no hacen, en rigor, una tarea esencialmente diferente de la que realiza quien se dedica a la Filosofía Antigua (o a cualquier período de la historia de la filosofía): tratar de hacer sentido del pensamiento de un autor sobre un problema filosófico determinado. Parece que el ocuparse de entender un texto más cercano en el tiempo, no obstante, tuviera algún tipo de legitimación intrínseca que el estudioso de Platón o de Numenio de Apamea debe forjar para sí. Esto a pesar de que, como señala perspicazmente Marcelo Boeri, ${ }^{3}$ si bien es cierto que alguien podría dudar del valor de la lectura de estos textos apoyado en el hecho de que el mundo de los griegos "ya no existe", también es cierto que en buena medida el mundo de hace cuarenta u ochenta años, sobre el que reflexionaron muchos de los más grandes filósofos del siglo XX, ya no es el mismo que el mundo en el que vivimos en muchos sentidos relevantes -baste pensar en la creciente aceleración en el ritmo de los avances científico-tecnológicos y de la transmisión y disponibilidad

\footnotetext{
${ }^{2}$ Quiero agradecer a Paloma Baño, Francisco Alonso, José Antonio Valdivia y Fabián Mié por leer este ensayo que hace las veces de presentación y por sus observaciones al texto. Naturalmente, no quiero sugerir con esto que ellos suscriban necesariamente todos mis puntos de vista.

${ }^{3}$ M. Boeri 2000, 132.
} 
de la información-. En este contexto no pretendo desplegar una apología extensa del valor del estudio académico de la Filosofía Antigua. Lo cierto es que no tengo demasiadas consideraciones de peso para agregar a las que ya han ofrecido algunos grandes filósofos y helenistas. ${ }^{4}$ Me gustaría simplemente esbozar algunas reflexiones sobre la base que ellos han sentado, para tratar de acercar al lector académico especializado y no especializado a este conjunto de artículos de alto rigor.

Creo que uno puede aproximarse al valor que tiene hoy día el estudio los textos de autores antiguos desde dos grandes vertientes: la que se refiere al contenido de esos textos, y la que se refiere a los aspectos formales, argumentativos o metodológicos que se encuentran en estos textos. En todos los casos, estas consideraciones suponen que quien se acerca a los antiguos lo hace lejos de una actitud reverencial o dogmática, es decir, lejos de una actitud que refrenda la creencia de que en ellos se encuentra algún tipo de verdad originaria, ante la cual sólo se puede prestar asentimiento una vez descubierta. Tampoco es una actitud genuinamente filosófica la que hace de estos textos un uso instrumental, es decir, la que acude a ellos para confirmar las propias opiniones acomodando los textos a ese efecto y, en el mismo trámite, le da a sus juicios la pátina de la autoridad de los antiguos. Me gusta recordar, a propósito de este uso de los antiguos, la genial irreverencia de Jacques Brunschwig 1994, 60: "Si para dar un sentido a nuestro trabajo sobre los pensadores del pasado no tenemos más recurso que insuflarles nuestra pequeña vida, hacerles abrazar nuestras causas (...) entonces, ¿para qué servirnos de ellos? Batámonos en persona, a cara descubierta y, de ser posible, dejemos a los muertos en paz.”

Más bien, hablo en estas páginas a, y de, quienes van a los textos antiguos con un interés filosófico; un interés que, además, sin descuidar el aspecto histórico, no se reduce a él. En otras palabras, las consideraciones que siguen sólo se entienden desde el supuesto de que una precondición para comprender genuinamente estos o cualquier texto filosófico es que quien se dirija a ellos lo haga siempre con un ojo puesto en el problema mismo que ellos discuten. ${ }^{5}$ Este modo de hacer hablar a los textos, de interpelarlos y de dejarse interpelar por ellos, conlleva necesariamente una toma de postura crítica respecto del modo en que el autor ilumina el problema en cuestión, y de la respuesta que da a ese problema. ${ }^{6}$ Se trata, en definitiva, de pensar problemas filosóficos con, y a partir de, los textos; y los artículos que integran este volumen son excelentes ejemplos de este tipo de ejercicio filosófico.

4 Véase especialmente Wolfgang Wieland 1988; Pierre Aubenque 1994; Jacques Brunschwig 1994; Marcelo Boeri 2000: Alejandro Vigo 2018.

${ }^{5}$ Una de las tantas enseñanzas que ha dejado W. Wieland 1988.

${ }^{6}$ Pueden verse las iluminadoras indicaciones al respecto de P. Aubenque 1994, quien se ocupa además del problema que naturalmente acecha tras esta postura, que es el del anacronismo. 
Considerando el asunto desde el punto de vista metodológico y argumentativo, primeramente, puede decirse que los textos antiguos suelen ser una fuente de inspiración para muchos filósofos contemporáneos, ${ }^{7} \mathrm{y}$ una instancia enormemente formativa para cualquier estudioso y estudiante de filosofía. Los filósofos de la Antigüedad despliegan, en su mayoría, estrategias argumentativas complejas y sofisticadas. En esta medida, sumergirse seriamente en estos textos constituye un aprendizaje privilegiado de un modo de hacer filosofía, i.e. de cómo analizar argumentalmente, plantear con claridad y discutir rigurosamente un problema filosófico y sus aristas.

Quiero reparar también, un poco menos brevemente, en el valor de los textos antiguos desde el punto de vista de su contenido, es decir, de los problemas filosóficos planteados (las más de las veces, inaugurados) por autores antiguos y de las respuestas que ellos mismos han ensayado frente a esos problemas que, también muchas veces, prefiguran los contornos de los debates en siglos posteriores y aún hasta nuestros días. En ese sentido suele emplearse hoy día el término "rehabilitación" para referirse a la búsqueda de respuestas y puntos de vista iluminadores en los autores antiguos por parte de los filósofos contemporáneos. No se trata tanto de detectar prefiguraciones rústicas, en un espíritu arqueológico, de teorías que luego toman formas nuevas y sofisticadas sobre bases completamente diferentes, sino de redescubrir aquellos problemas y respuestas cuya vigencia sigue siendo tan actual como en el momento de su planteo, y que son adoptados desde nuevos aparatos conceptuales y contextos de reflexión. Ejemplos paradigmáticos de esto son el problema de la contraposición entre el bien real y el bien aparente planteado por primera vez por el Sócrates platónico, ${ }^{8}$ y la enorme influencia de Aristóteles en la filosofía de la acción dentro de la tradición analítica del siglo XX, no sólo en la explicación de la acción racional -el silogismo práctico formulado por primera vez por Aristóteles es reinstaurado como la base del modelo explicativo de la acción tanto por Elisabeth Anscombe como por Donald Davidson-, ${ }^{9}$ sino incluso en el análisis teórico de ciertos fenómenos de irracionalidad interna, como la debilidad de la voluntad. ${ }^{10}$

Más aún, hay casos en que las respuestas de los antiguos a determinados problemas aún vigentes, tras haber sido olvidadas largo tiempo por azares de la historiografía, revisten el carácter de genuinas novedades filosóficas al ser redescubiertas desde contextos problemáticos diferentes a los que les dieron

${ }^{7}$ Sellars 2003, 24, señala que el aspecto argumentativo de la filosofía antigua la acerca especialmente a la filosofía angloamericana académica contemporánea.

${ }^{8}$ Ver M. Boeri 2000, 148-152 para una informativa exposición del problema en los diálogos platónicos y de su vigencia en la filosofía contemporánea.

${ }^{9}$ Véase G. E. M. Anscombe 1963 y D. Davidson 1963.

${ }^{10}$ Véase D. Davidson 1979. Para una exposición más extensa de este punto véase A. Vigo 2018, 137-139. 
origen. Es este el caso, por ejemplo, de las antiguas éticas de la virtud de origen platónico y aristotélico, que aparecen a mediados del siglo XX como una alternativa frente a lo que se percibía como un agotamiento de los modelos deontológico y utilitarista que dominaban entonces la escena del debate ético. ${ }^{11} \mathrm{La}$ vitalidad de la ética de la virtud ha sido creciente desde entonces, dentro y fuera de la filosofía, al punto de exceder los límites que la separaban de otros modelos supuestamente heterogéneos. Tal es así que hoy día existen no sólo éticas de la virtud de corte neoaristotélico, ${ }^{12}$ sino que incluso se habla de éticas de la virtud kantianas y utilitaristas. ${ }^{13}$ En todos estos casos, la referencia a los textos platónicos y, en especial, aristotélicos de filosofía práctica, que constituyen el planteo clásico más maduro de una ética de la virtud, es ineludible y recurrente. ${ }^{14}$ Dentro de la discusión contemporánea, y fuera de la ética de la virtud, la ética de Aristóteles es también una de las fuentes de la que abreva expresamente la teoría normativa de Christine Korsgaard; e incluso es fuerte inspiración de posturas de corte no-naturalista (un caso paradigmático es John McDowell).

El de la filosofía práctica, con todo, no es el único ámbito en que la relectura de los antiguos resulta productiva. Un caso similar a este está configurándose en el terreno de la metafísica analítica de los últimos años de nuestro siglo, en especial en el terreno de la filosofía de la mente, en que el hylemorfismo aristotélico resurge con fuerza frente a modelos reductivos materialistas derivados del dualismo cartesiano. ${ }^{15}$ Se trata de la llamada metafísica neoaristotélica de corte analítico. ${ }^{16}$ Incluso, en relación con la búsqueda de alternativas filosóficas frente al materialismo contemporáneo, vale traer a colación el polémico libro de Thomas Nagel 2012, que defiende la necesidad de incorporar explicaciones de tipo teleológico no providencialista para dar cuenta de la naturaleza en su conjunto, incluyendo la evolución y el origen de la vida. No es difícil

${ }^{11}$ En este plano se considera decisivo y pionero el trabajo de G. E. M. Anscombe 1958, que diagnosticaba que la idea de obligación moral requerida por la deontología y el utilitarismo carece de sentido en el marco de una moral secular. En este escenario, la autora propone que la moral debe volver a la idea de virtud tal como la propusieron Platón y Aristóteles.

${ }^{12}$ Cf. por ejemplo Rosalind Hursthouse 1999, Julia Annas 2011. El naturalismo moral de Philippa Foot, si bien tiene apoyo en la ética de la virtud de Tomás de Aquino, es con todo fuertemente deudor de ciertas ideas y esquemas teóricos no sólo de la ética sino de la filosofía natural de Aristóteles (en especial cf. Foot 2001).

${ }^{13}$ Para un panorama sucinto de la paleta de posturas de raíz platónico aristotélica que hoy día se agrupan bajo las éticas de la virtud, véase Roberts 2017.

${ }^{14}$ Cf. R. Crisp - M. Slote 1997.

15 David Charles 2008, muestra en qué medida el hylemorfismo aristotélico puede constituir una alternativa superadora del dualismo cartesiano; y en el terreno de la filosofía de la mente analítica contemporánea William Jaworski 2016, por caso, ofrece una solución hylemorfista al problema de la relación mente-cuerpo.

16 Cf. M. Boeri - Y. Kanayama 2018, 1-10, y los volúmenes de artículos editados por Edward Feser 2013 y Tuomas Tahko 2011. Para la actualidad de Aristóteles en la ontología tanto analítica como continental del siglo XX, cf. también A. Vigo 2018, 128-136. 
encontrar cierta comunidad de inspiración entre la propuesta de Nagel y algunos aspectos de la teleología natural aristotélica. ${ }^{17}$

La idea de que uno puede encontrar en los pensadores del pasado combustible para las propias elaboraciones filosóficas era compartida por Platón y por Aristóteles, que plantean su filosofía de modo consciente en discusión con quienes los antecedieron. Hoy día no sólo tiene lugar esta vertiente dialógicopolémica con el pasado, sino incluso la inspiración positiva en pensadores que reflexionaron hace mucho tiempo acerca de los mismos problemas (o de problemas muy similares) que enfrentamos hoy. La filosofía, tal parece, no puede avanzar sino nutriéndose de sí misma y en discusión con su propia tradición. Cuando no lo hace, ella ciertamente es presa del riesgo de repetir viejas doctrinas como si fueran novedades - como una suerte de Pierre Menard involuntario- y, en cierto modo, se resigna a convertir en un monólogo ingenuo lo que podría ser un diálogo enriquecedor con estructuras conceptuales ya ganadas en el pasado. ${ }^{18}$ Pero no sólo por esta razón el estudio de la historia de la filosofía cobra un valor crucial.

Soy consciente de que probablemente, no sin cierto recelo suspicaz, se imagine que el apego excesivo (o quizá reverencial) al estudio de la historia de la filosofía sea a la vez producto y causa de una visión conservadora de la disciplina, en la medida en que conduce a comprendernos a nosotros mismos y a nuestra actividad filosófica en el marco de una tradición que podría considerarse, por diversas razones, superada, deficiente o negativa en algún sentido. Este tipo de visiones, a veces, transforma una sospecha heredada acerca de la "tradición" filosófica en una razón para sentirse eximido de tomarse el trabajo de conocerla. Permítaseme decir esto de otra manera.

El modo en que una cultura se comprende a sí misma viene determinado en parte por aquello que define como sus "clásicos", y esto vale tanto en el sentido trivialmente positivo como también en el sentido negativo; es decir, el sentido que se manifiesta allí donde los clásicos son aquello frente a lo cual cierta cultura se define por oposición o frente a lo cual se autocomprende como superadora. Es, pues, tan significativo conocer a los clásicos para aprender de ellos como para distanciarse de ellos críticamente. Esta es una lección que algunos grandes filosófos del siglo XX conocían muy bien y, por eso, de entre ellos, quienes se separan críticamente de la tradición filosófica lo hacen siempre

${ }^{17}$ Ejercicios ejemplares de actualizaciones creativas de la filosofía antigua por parte de especialistas (o scholars), pueden encontrarse en A. Vigo 2006 -quien relee la Física de Aristóteles en términos de las estructuras ontológicas del ente natural que son develadas por medio de una concepción metódica que el autor llama, con buenas razones, fenomenológica- y, en su conjunto, en la colección de artículos contenida en Sarah Broadie 2007.

${ }^{18} \mathrm{~J}$. A. Valdivia 2018 muestra ejemplarmente las consecuencias teóricas este tipo de desconocimiento de la historia de la filosofía (en particular, de discusiones gnoseológicas en la Filosofía Medieval) en la teoría de la percepción de J. Searle. 
desde esa tradición filosófica que buscan superar. Ahora bien, pretender superar una tradición filosófica es un asunto diferente de declarar su irrelevancia (más bien presupone lo contrario). Lamentablemente, no siempre ocurre esto en el contexto de la reflexión filosófica actual (incluso dentro de la academia) con quienes se declaran seguidores de estos grandes filósofos del siglo XX. Más bien, seducidos por la retórica de la superación de la tradición, muchos cometen el error de sentirse eximidos de conocer de primera mano esa tradición, lo cual los lleva, o bien a desmarcarse de una supuesta tradición que en verdad se construye como un hombre de paja, o bien - a veces como resultado de lo anteriora entender que en esta superación o distanciación propuesta se esconde un diagnóstico de abandono. El resultado es un progresivo, y autolegitimado, olvido (a nivel colectivo) e ignorancia (a nivel individual) de aquello frente a lo cual se está, supuestamente, tomando postura, lo cual deriva en una repetición acrítica de la mera gestualidad de una rebelión que, sin embargo, se va vaciando progresivamente de sentido al desdibujar casi completamente su blanco.

La filosofía antigua, quizá por la dificultad que conlleva su estudio, suele ser una de las primeras víctimas de esta vocación pseudosuperadora que se cultiva en algunos ámbitos académicos. La dificultad a la que me refiero se debe en buena medida al hecho de que los textos de los antiguos están escritos en lenguas que hoy día, en muchos países de Latinoamérica y de otras partes del mundo, ya no se enseñan o se enseñan cada vez menos: el latín y el griego antiguo. A ello se suma el hecho de que el mundo que ella describe es, como decía más arriba, un mundo diferente del nuestro, para acercarse al cual es necesaria una formación adecuada y una destreza interpretativa específica. En otras palabras, la distancia histórica que nos separa de los filósofos antiguos exige al estudioso un especial esfuerzo de comprensión y una enorme prudencia hermenéutica para no proyectar de modo acrítico los presupuestos de la propia visión de mundo a un texto escrito desde una visión de mundo, en ocasiones, muy diferente. La distancia entre las cosmovisiones que aflora de este modo, con todo, constituye uno de los aspectos filosóficamente más fructíferos que arroja el estudio de la filosofía de este período. Constatar que los antiguos se plantearon problemas similares a los nuestros pero los resolvieron de modo completamente diferente al que nosotros esperaríamos, o bien que no se plantearon problemas o aristas de problemas que para nosotros son cruciales e ineludibles, o bien que en algunos casos comprendían la actividad filosófica misma de un modo diferente al nuestro, nos ayuda a comprender no sólo a los antiguos, sino a nosotros mismos. Estas distancias ponen de relieve de modo especialmente claro, para un lector filosóficamente atento y metodológicamente maduro, los presupuestos del propio momento histórico, filosófico y cultural. Este tipo de autoconocimiento crítico es, en definitiva, parte de lo que se pierde a partir del abandono del estudio de la historia de la filosofía, no sólo en el período antiguo. 


\section{SOBRE LOS TEXTOS QUE INTEGRAN ESTE VOLUMEN}

Los siete artículos que integran este número especial recorren diversos problemas discutidos por autores de la Antigüedad, desde Platón, hasta los comienzos del neoplatonismo. No obstante, como podrá comprobar el lector, la mayoría de los textos está dedicada a la filosofía de Aristóteles.

El artículo de Ivana Costa está dedicado a reflexionar sobre el uso de la ficción en Platón, un uso que la autora pone en conexión y analiza desde la revalorización en teorías contemporáneas del papel de la ficción en filosofía como un elemento que contribuye a forjar racionalidad. Costa se detiene, primero, en el análisis de la evolución semántica del término griego utilizado para designar las ficciones como un relato modelado o inventado (sentido que aparece en Platón y Aristóteles), a partir de un sentido original que tiene su origen en el modelado de lo corpóreo. Finalmente, la autora muestra cómo este tipo de ficciones tiene un rendimiento filosófico en especial en Platón; no sólo en el caso de ficciones teóricas (como la teoría de las ideas en el Fedón), sino incluso y más específicamente en el caso de las ficciones políticamente funcionales presentadas en República y Leyes como relatos que es útil contar a los ciudadanos de las respectivas ciudades ideales, en vistas de su valor práctico respecto de la virtud.

En su sesudo artículo, Paloma Baño analiza uno de los problemas que presenta la compleja concepción aristotélica del tiempo en Física IV 10-14. Allí, Aristóteles presenta lo anterior y lo posterior como parte de la propia definición del tiempo. No obstante, las nociones de "anterior" y "posterior" no pueden ser primariamente temporales, en la medida en que, en tal caso, la definición aristotélica del tiempo sería circular. En otras palabras, para que la definición del tiempo de Aristóteles pueda ser tomada en serio, es necesario interpretar el par anterior-posterior en un sentido no temporal. Esto sin duda constituye un desafío filosófico en sí mismo. La autora ofrece, a lo largo de las páginas que componen el artículo, una interpretación plausible que muestra cómo Aristóteles concibe efectivamente un anclaje en fenómenos no temporales para la anterioridad y la posterioridad. Para ello, ella examina en qué sentido debe comprenderse la tesis que sostiene la vinculación entre tiempo, movimiento y magnitud (219a10-19), y logra mostrar cómo la antero-posterioridad que se halla en la magnitud y que es condición de posibilidad de la antero-posterioridad temporal no presupone, sin embargo, el aspecto temporal de "anterior" y "posterior”, sino que consiste en la heterogeneidad de posiciones de un móvil.

El artículo de Fabián Mié está enfocado en el problema de la unidad de la sustancia compuesta en la Metafísica de Aristóteles. El autor aborda en particular el papel de los capítulos de Metafísica H1-5 de cara a la elaboración de la concepción modal de la sustancia compuesta en H6. El papel causal que juega respectivamente la forma y la materia es examinado en estas páginas de 
modo exhaustivo a partir de una lectura minuciosa de los primeros cinco capítulos de $\mathrm{H}$ que permite develar no sólo sofisticadas estructuras argumentativas en este texto, sino además refinadas construcciones conceptuales que buscan resolver algunos problemas que el último capítulo del libro $\mathrm{Z}$ había dejado irresueltos, en particular los referidos al papel causal de la forma y a la unidad de la sustancia material. El lector encontrará especialmente interesante el análisis del rol causal de la materia en sus diferentes niveles de determinación, que Mié ofrece a partir de su lectura de H4-5; un análisis que promete frutos más allá de este texto, por ejemplo, para la lectura de algunos problemas clásicos que enfrenta el hylemorfismo (o que los intérpretes de Aristóteles han creído encontrar en él, como el llamado problema de Ackrill).

El siguiente artículo está a cargo de Alberto Ross, quien aborda el problema de la teleología que rige el ámbito de las entidades naturales compuestas de forma y materia, y, en especial, la relación de esta teleología natural con la sustancia eterna e inmóvil de Metafísica $\Lambda 6-7$. La lectura del autor es más bien deflacionaria, en la medida en que sostiene que el primer motor inmóvil de la Metafísica no cumple un papel causal fuerte respecto de la teleología natural. Esto debe entenderse en el siguiente sentido: el primer motor permite explicar la eternidad del movimiento y del tiempo en la naturaleza entendida como totalidad; pero no cumple un papel causal en relación con la naturaleza de las entidades particulares. Ross muestra que en el tipo de causalidad cosmológica que ejerce este primer motor, por lo tanto, no hay involucrado componente alguno de tipo providencialista o iusnaturalista.

El artículo de Viviana Suñol gira alrededor de las funciones que Aristóteles asigna a la música en su Política VIII 3, 5-7. Teniendo en cuenta que la mousikē es el núcleo del programa educativo que Aristóteles diseña en dicha obra para el mejor régimen, y que esta educación operada por la mousikē remite especialmente al componente psicofísico emocional de la virtud, resulta de crucial importancia determinar el orden jerárquico de las funciones que el filósofo atribuye a esta disciplina. Suñol se enfoca particularmente en el papel de la katharsis, una de las potencias de la música. La autora muestra que no se puede asociar la katharsis rígidamente ni con la función de relajación (o lúdica) ni con la función educativa de la mousikē, sino que la posición de Aristóteles respecto de la katharsis parece oscilar entre ambas funciones. Finalmente, Suñol considera el relevante asunto de la katharsis en relación con la educación moral, y muestra que, lejos de identificarse con la educación emocional tendiente a la virtud moral, la katharsis presupone dicha educación.

Diego Zucca ofrece en su lúcido artículo una lectura global del texto De Anima III 4 de Aristóteles dedicado al intelecto (nous), en la que reconstruye una estructura argumental deductiva que estructura el capítulo. De acuerdo con la reconstrucción de Zucca, las características del nous se siguen unas de otras 
necesariamente y según una lógica deductiva, en lugar de ser presentadas de modo rapsódico por Aristóteles. Según la lectura propuesta por el autor, Aristóteles deriva lógicamente la separabilidad del nous a partir del hecho de que él no está mezclado con el cuerpo. De la separabilidad del nous Aristóteles deriva a su vez la espontaneidad, y de la espontaneidad la autointelección. Estas implicaciones son reconstruidas a partir del planteo y discusión de las dos aporías al interior del capítulo III 4, cuyo valor teórico y metodológico es puesto así de relieve por Zucca.

El último artículo de este volumen, de Gabriela Müller, nos deposita ya en el período previo al surgimiento del neoplatonismo. Aquí encontramos un estudio de los conceptos de alma y cuerpo y del problema de su relación en la filosofía de Numenio de Apamea, un platónico del siglo II d. C. La filosofía de Numenio se conserva sólo en fragmentos y testimonios de diversas fuentes, que Müller analiza críticamente. La postura de Numenio es reconstruida sobre el trasfondo de su polémica con el materialismo estoico, un horizonte contra el cual el filósofo de Apamea moldeó su propia concepción cosmológica y antropológica. En ese marco, Numenio postula para el alma dos funciones aparentemente difíciles de compatibilizar: la de ser principio de estabilidad y principio del movimiento. Müller explica cómo, en verdad, estas dos funciones del alma están lejos de ser incompatibles. Para ello, la autora muestra que Numenio concibe dos tipos de movimiento: uno que garantiza la estabilidad, el orden y la racionalidad de lo real, y el otro que implica irracionalidad y desorden.

No puedo terminar estas páginas sin expresar mi sincero agradecimiento a los autores que han colaborado con su trabajo en este magnífico volumen, por su talento, por su entusiasmo, por su buena disposición y por su inquebrantable paciencia con las exigencias y sugerencias de esta editora. Dejo presentado este conjunto de estudios con la esperanza de que ellos despierten el interés del estudioso de la filosofía antigua, y de que el lector especializado y no especializado encuentre en ellos el mismo disfrute que me ha tocado experimentar al leerlos.

\section{BIBLIOGRAFÍA}

Annas, J. 2011. Intelligent Virtue, Oxford: OUP.

Anscombe, G.E.M. 1963. Intention, Oxford: Blackwell.

Anscombe, G.E.M. 1958. "Modern Moral Philosophy”, reimpreso en R. Crisp M. Slote (eds.), Virtue Ethics, Oxford: OUP, 1997; 26-44.

Aubenque, P. 1994. "Sí y no", en B. Cassin (ed.), Nuestros Griegos y sus modernos. Estrategias contemporáneas de apropiación de la Antigüedad, Buenos Aires: Manantial; 19-31.

Boeri, M. 2000. “¿Por qué ocuparse de filosofía antigua hoy?”, Kléos 4: 131-153. 
Boeri, M. - Kanayama, Y. 2018. "General Introduction", en M. Boeri - Y. Kanayama - J. Mittelmann (eds), Soul and Mind in Greek Thought. Psychological Issues in Plato and Aristotle, Cham: Springer; 1-15.

Broadie, S. 2007. Aristotle and Beyond, Cambridge: CUP.

Brunschwig, J. 1994. "Hacer historia de la filosofía, hoy", en en B. Cassin (ed.), Nuestros Griegos y sus modernos. Estrategias contemporáneas de apropiación de la Antigüedad, Buenos Aires: Manantial; 53-71.

Charles, D. 2008. "Aristotle's Psychological Theory”, Proceedings of the Boston Area Colloquium of Ancient Philosophy 24 (1): 1-49.

Crisp, R. - Slote, M. 1997. "Introduction", en R. Crisp - M. Slote (eds.), Virtue Ethics, Oxford: OUP; 1-25.

Davidson, D. 1963. "Actions, Reasons and Causes", en D. Davidson, Essays on Actions and Events, Oxford: Clarendon Press, 1980; 3-19.

Davidson, D. 1979. "How is Weakness of the Will Possible?", en D. Davidson, Essays on Actions and Events, Oxford: Clarendon Press, 1980; 21-42.

Feser, E. (ed.) 2013. Aristotle on Method and Metaphysics, New York: Palgrave Macmillan.

Foot, Ph. 2001. Natural Goodness, Oxford: OUP.

Hursthouse, R. 1999. On Virtue Ethics, Oxford: OUP.

Jaworski, W. 2016. Structure and the Metaphysics of Mind. How Hylomorphism Solves the Mind-Body Problem. Oxford: OUP.

McDowell, J. 1998. "Two sorts of Naturalism", en J. McDowell, Mind, Value, and Reality, Cambridge MA: Harvard University Press; 167-197.

Nagel, Th. 2012. Mind and Cosmos. Why the Materialist Neo-Darwinian Conception of Nature is Almost Certainly False, Oxford: OUP.

Roberts, R. 2017. "Varieties of Virtue Ethics", en D. Carr - J. Arthur - K. Kristjánsson (eds.), Varieties of Virtue Ethics, New York: Palgrave Macmillan; 17-34.

Sellars, J. 2003. "Teaching Ancient Philosophy", Philosophical and Religious Studies-Subject Centre of the Learning and Teaching Support Network Journal 2 (2): 23-49.

Tahko, Th. (ed.) 2011. Contemporary Aristotelian Metaphysics, Cambridge: CUP.

Valdivia, J. A. 2018. “Para qué sirve la historia de la filosofía? A propósito de la teoría de la percepción de John R. Searle”. Pensamiento 74 (282): por aparecer.

Vigo, A. 2006. “¿Está obsoleta la Física de Aristóteles?”, Estudios Públicos 102: 43-67.

Vigo, A. 2018. “Aristóteles y la filosofía actual”, Estudios Públicos, 151: 123-147.

Wieland, W. 1988. "La actualidad de la filosofía antigua", Méthexis I: 3-16. 